\title{
An Ultrasonic Motor Using Bending Cylindrical Transducer Based On PZT Thin Film
}

\author{
Takeshi MORITA, Minoru KUROSAWA and Toshiro HIGUCHI \\ Dept of Precision Machinery Engineering, Faculty of Engineering, University of Tokyo \\ 7-3-1 Hongo, Bunkyo-ku, Tokyo 113, JAPAN \\ Phone;+8133812 2111 ext.6448, \\ Fax;+81358006968
}

\begin{abstract}
Ultrasonic motors have a distinguished performance of high torque. Especially, it seems that cylinder type ultrasonic motors are superior to disk or ring type ultrasonic motors in sense of high torque application. For miniaturization of a motor size, simple construction of an ultrasonic transducer become effective. In this work, we propose an ultrasonic motor using bending cylindrical transducer based on PZT thin film on side wall of a small tube. The transducer of the ultrasonic motor is composed of short cylindrical titanium pipe, PZT thin film and electrodes. The titanium pipe is introduced for a base material to deposit PZT thin film by hydrothermal method. The thickness of film is about from 7 to $9 \mu \mathrm{m}$. The electrodes are divided into 4 parts in circumferential direction to excite degenerated bending vibration mode with two RF driving electrical sources. A rotor loaded on the transducer turned around in the intended direction.The maximum revolution speed was 295 rpm.
\end{abstract}

\section{INTRODUCTION}

Recently, the study of micro electro mechanical systems have been developed rapidly and their achievement become a center of attraction.

To realize these systems, it is essential to miniaturize such as actuators, gears, sensors and so on. Particularly, the complexed structure of actuators make it difficult to fabricate.

In small dimensions, electrostatic force has advantage, so that various electrostatic motors has been investigated and got excellent result[1],[2]. Generally, electrostatic motors obtain low torque and might require gear boxes to assemble a system. Compared to electrostatic motors, ultrasonic motors indicate the possibility of high torque because piezoelectro force is very large.

Micro ultrasonic motors which have been already reported were all flat profile[3], [4]. However, for a purpose of a high torque motor, cylindrical motors might be superior to the flat one. In a cm-order diameter, it is already demonstrated that ultrasonic motors generate high torque[5],[6], and the advantage will be preserved at mm-order and sub mm-order diameter motor. So in this study, we adopted the cylindrical shape.

Moreover, it should be exhausted that ultrasonic motors have simple construction and no requirement for bearing. Particularly, utilizing lead zirconate titanate (PZT) thin film which has been deposited on base metal, the structure of our motor has remarkable simple construction.

\section{Structure}

The stator transducer of our micro ultrasonic motor is cylindrical shaped and $2.4 \mathrm{~mm}$ in diameter, $10 \mathrm{~mm}$ in length and $0.25 \mathrm{~mm}$ wall thick. It composes of titanium tube and four electrodes which sandwich PZT thin film as shown in Fig. 1. The PZT thin film is deposited by hydrothermal method and it's thickness is about from 7 to $9 \mathrm{~mm}$. The polarization of the PZT film is the thickness direction. The detail of hydrothermal method will be described later.

Four copper wires $0.1 \mathrm{~mm}$ in diameter were fixed on each electrodes about $2.2 \mathrm{~mm}$ above from the bottom surface to prevent disturbing the vibration. The role of four wires were not only supplying driving source but also supporting the stator transducer.

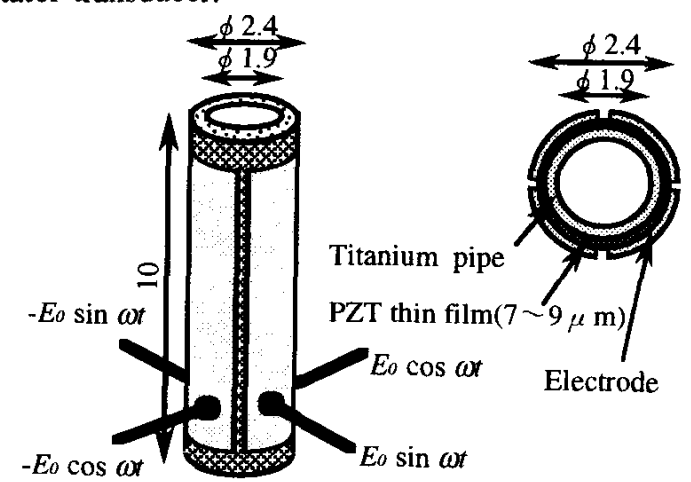

Fig. 1. Construction of micro ultrasonic motor 


\section{Principle}

As the same as the other mode rotation type ultrasonic motors, this motor utilized the traveling wave on the end surface of the stator transducer. The vibration of the stator transducer is transformed to rotation motion through the frictional force.

As described before, the polarization of PZT was thickness direction. With one RF electrical source to one pair of electrodes facing each other, the fundamental bending vibration of the stator transducer is excited with piezoelectro lengthextentional effect as shown in Fig. 2.

By using two RF electrical sources which have 90 degrees phase difference each other, the rotation mode of the stator transducer is excited as shown Fig. 3. It should be noted that one wavelength traveling wave is generated on the end surface. So the rotor loaded on the transducer turns around in the indicated direction.

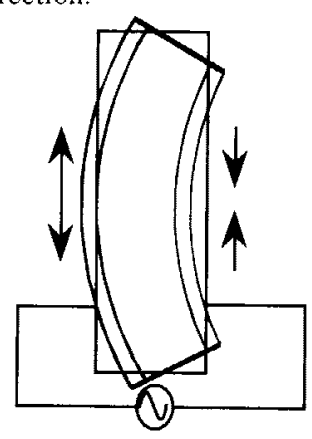

Fig. 2. Excitation of vending vibration.

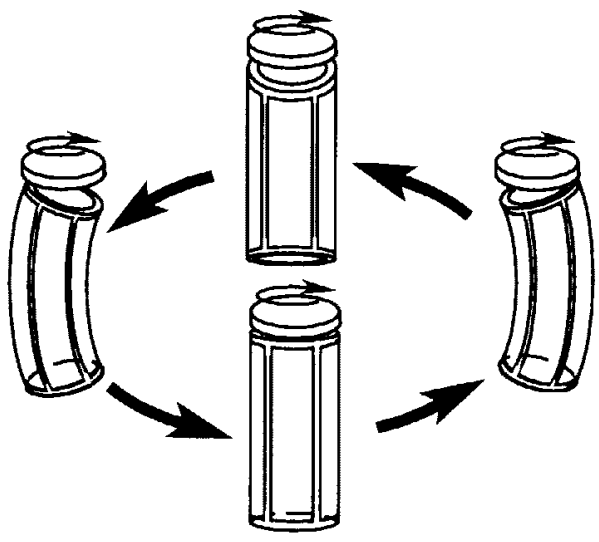

Fig. 3. Principle of micro ultrasonic micro motor using two bending modes.

\section{ViBRATION MODE}

To obtain enough vibration amplitude, it is important to make the stator transducer vibrate at a resonance frequency. The resonance frequency is calculated by following the Timoshenko differential equation [7].

$$
E I \frac{\partial^{4} \xi}{\partial x^{4}}+\rho A \frac{\partial^{2} \xi}{\partial t^{2}}-\rho I\left(1+\frac{E}{k G}\right) \frac{\partial^{4} \xi}{\partial x^{2} \partial t^{2}}+\frac{\rho^{2} I}{k G} \frac{\partial^{4} \xi}{\partial t^{4}}=0
$$

where

$E$ : Young's modules of titanium $\left(1.1 \times 10^{11 \mathrm{~N}} / \mathrm{m}^{2}\right)$, I: polar moment of inertia of area $\left(9.75 \times 10^{-13} \mathrm{~m}^{4}\right)$, $\rho$ : density of titanium substrate $\left(4.51 \times 10^{3} \mathrm{~kg} / \mathrm{m}^{3}\right)$, $A$ : cross section of the stator $\left(1.69 \times 10^{-6} \mathrm{~m}^{2}\right)$, $\kappa$. an adjustment coefficient (10/9),

$l$ : length of stator $\left(1.0 \times 10^{-2} \mathrm{~m}\right)$,

$G$ : modules of transverse elasticity $\left(3.98 \times 10^{10} \mathrm{~N} / \mathrm{m}^{2}\right)$.

The boundary conditions are written as follows:

$$
\frac{d^{2} \xi(0)}{d x^{2}}=\frac{d^{3} \xi(0)}{d x^{3}}=0 \quad, \quad \frac{d^{2} \xi(l)}{d x^{2}}=\frac{d^{3} \xi(l)}{d x^{3}}=0 .
$$

Using the solution from the equation, the resonance frequencies are expressed by

$$
f_{r}=\frac{1}{2 \pi}\left(\frac{\alpha}{l}\right)^{2} \sqrt{\frac{E I}{\rho A}}\left[1-\frac{I}{2 A}\left(\frac{\alpha}{l}\right)^{2}\left(1+\frac{E}{k G}\right)\right] .
$$

Our expected mode is first mode, so that the frequency parameter $\alpha=0.470$. This equation shows the resonance frequency $\mathrm{f}_{\mathrm{r}}=114 \mathrm{kHz}$.

Moreover, the vibration mode is expressed by

$$
\xi=C\left(\sin \frac{\alpha}{2} \cosh \frac{\alpha}{l} x-\sinh \frac{\alpha}{2} \cos \frac{\alpha}{l} x\right)
$$

where $\mathrm{C}$ is constant. Figure 4 shows expected mode of our stator. This graph indicates that this mode has two nodal points $(x=0.281$ and $x=-0.281)$ which do not vibtate in radial direction.

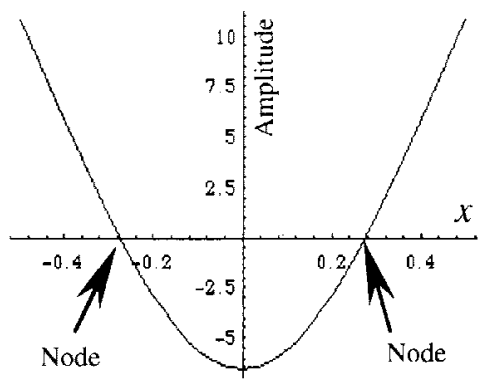

Fig. 4. Simulation which shows amplitude in axial direction on side surface. 


\section{Fabrication of Stator Transducer}

Fabrication process of the stator transducer has four steps. Each step is indicated in Fig. 5. They are,

(1) cut the titanium pipe in appropriate length and polish the side wall,

(2) deposit the PZT film by the hydrothermal process, and after this process, polish the end surfaces,

(3) paint the silver past for driving electrodes,

(4) connect the copper wire to supply the RF electricity at one of the nodal points as described before.

When this motor was operating, the top surface clearness was an important factor. So before operating, we wiped the contact surface of stator and rotor with alcohol.

\section{Hydrothermal method}

There are several ways to deposit piezoelectric thin film. Sol-gel process[8] is rather popular. This method has already tried to make the micro ultrasonic motor. But a problem of this method is a difficulty to get thick material such as $10 \mu \mathrm{m}$. In order to take out enough force, thick film is required for a stator transducer. Accordingly, we have introduced a hydrothermal synthesis[9] to deposit PZT film.

This method is carried out in liquid, hence the shape of a transducer is rather free. The required materials are solution of $\mathrm{Pb}\left(\mathrm{NO}_{3}\right)_{2}, \mathrm{ZrOCl}_{4}$, KOH and a solid titanium substrate. The titanium, a component of PZT, is supplied from the substrate. These materials were kept at 160 degrees for 48 hours in an autoclave whose capacity was $30 \mathrm{ml}$. The atmospheric pressure was about $6 \mathrm{~atm}$. To avoid the pin hole uncoated area, the autoclave was rotated during the process at several revolution per minutes.

The PZT film was successfully deposited on the curved side wall of the titanium pipe. The thickness of the film was about 7 to $9 \mu \mathrm{m}$. To confirm that the film was actually PZT, a $20 \mu \mathrm{m}$ titanium flat foil was also put in the autoclave to examine XRD test afterward. The result is shown in Fig.7.

Beside enough thickness, the characteristics of PZT film deposited by the hydrothermal method is high linearity of electro-field-induced displacement [9]. And more distinctive feature is that automatical polarization during the synthesis process, therefor, the actuator may have superior linearity. The high linearity suggests possibility of low mechanical vibration loss.

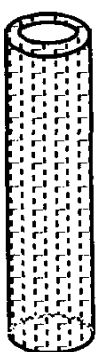

(1)

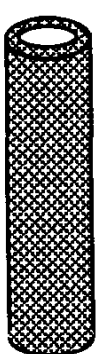

(2)

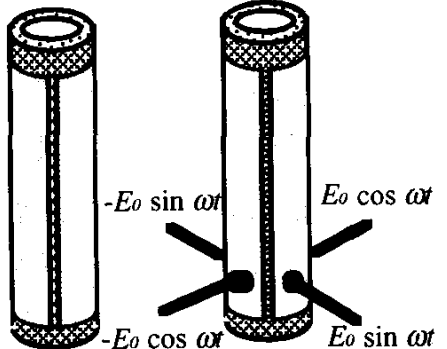

(4)
Fig. 5. Process of ultrasonic micro motor.

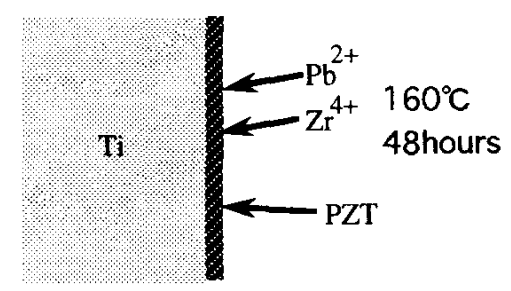

Fig. 6. Scheme of the process of PZT thin film.

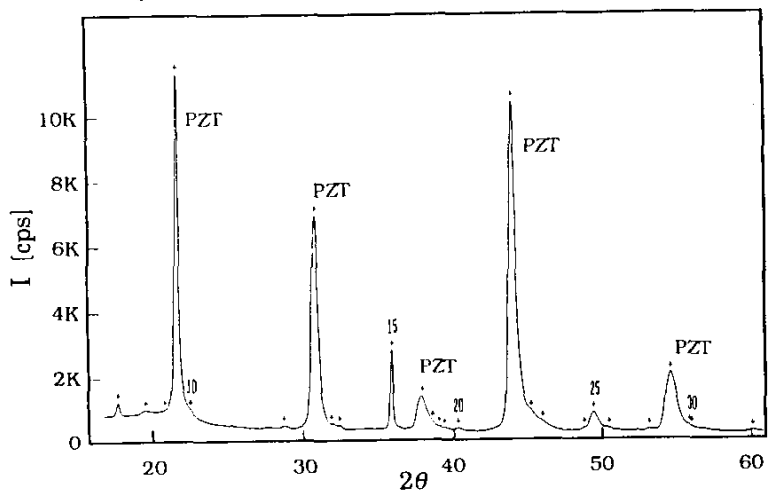

Fig.7. XRD pattern of thin film deposited on $\mathrm{Tj}$ substrate by hydrothermal method.

\section{Experiment}

To ascertain the properties of vibration, we measured the resonance frequency, vibration mode, end surface vibration distribution and so on by monitoring the vibration velocity using Laser Doppler Vibrometer.

In accordance with the principle, two RF 
power sources are required to excite the expected degenerated vibration. The circuit connections is shown in Fig. 8.

\section{Resonance frequency}

As shown in Fig. 9, projecting the laser vertical to the top of the side wall, we measured the relationship of the driving frequency to the vibration amplitude. The driving voltage was kept $25 \mathrm{Vp}-\mathrm{p}$ (in following measurements it was maintained).

As shown in Fig. 10, the resonance frequency was about $106 \mathrm{kHz}$. From the frequency response, the mechanical $Q$ factor was about 200 . Due to the large loss of the electrodes and the wire connection, the mechanical loss might be increased. In a result, rather high driving voltage was required to get enough vibration amplitude.

\section{Vibration mode}

The next interest is vibration mode. The measurement method was almost similar to mentioned above, except that the spot of the laser was traversed from the bottom to the top of the side wall. The coordinate is shown in Fig. 11; in short, at the bottom $x=0$ and at the top $x=10$. At several point the amplitude was measured as shown in Fig. 12.

The result indicates that the stator transducer had bending vibration mode as expected and there were two nodal positions.

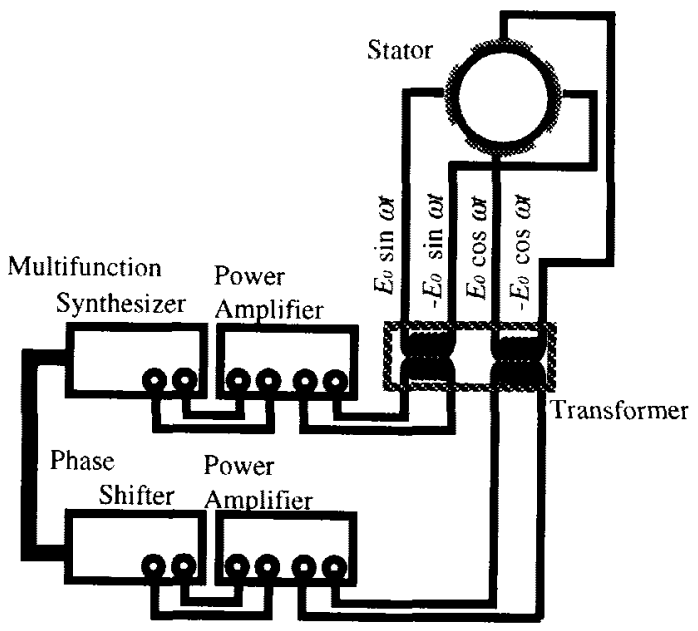

Fig. 8. Circuit connection for excitation of degenerated vibration mode.

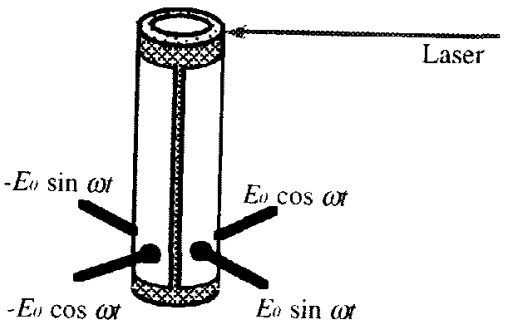

Fig. 9. Measured point of vibration amplitude.

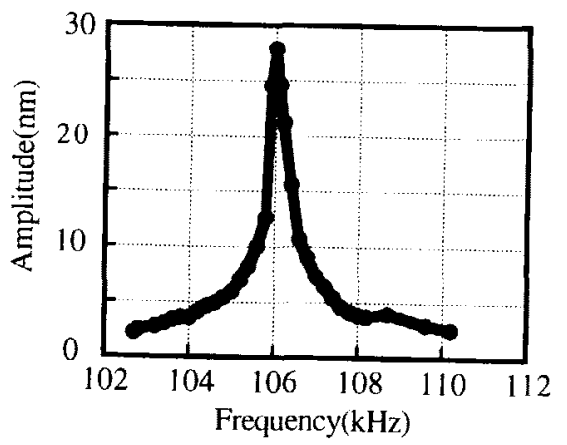

Fig. 10 Vibration amplitude of stator versus applied frequencies.

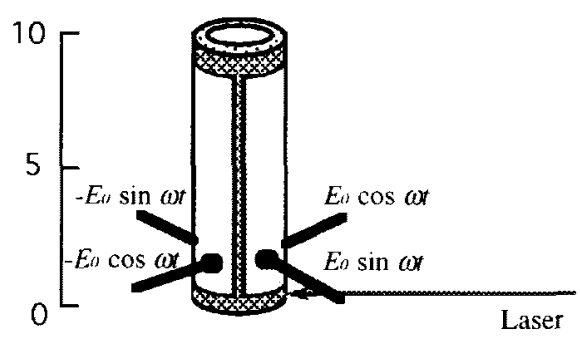

Fig. 11 Measured point of vibration mode.

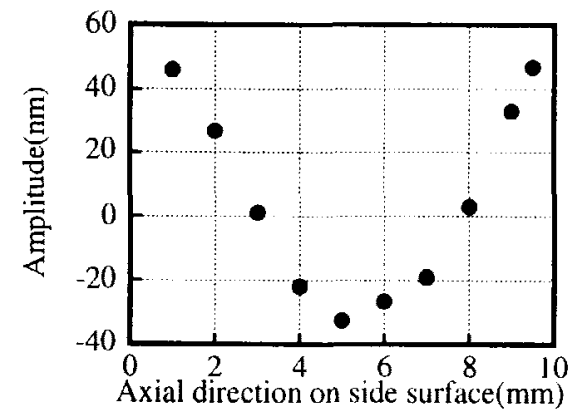

Fig. 12 Amplitude distribution on direction on side surface 


\section{Vibration distribution of end surface}

Only this experiment, one RF power source was supplied to one pair of electrodes facing each other (resonance frequency was $109 \mathrm{kHz}$ ). In such situation, standing wave should be generated on end surfaces.Projecting laser to the top surface, we measured the vibration displacement distribution of the top surface.

As shown in Fig. 13, one of four wires is regarded as a reference point of $\theta$. And $\theta$ is defined counterclockwisely. Shifting the spot of laser, the vibration amplitude was measured at each $\theta$ point.

The result which is shown in Fig. 14 indicates that one wavelength standing wave was excited along circumferential direction as expected. With one more RF power source which phase difference is 90 degrees, the vibration may be degenerated and traveling wave should be excited.

\section{Excitation of traveling wave}

We confirmed whether traveling wave was excited with two RF power sources. In the same way mentioned above, $\theta$ was defined and at each $\theta$ point, the axial direction vibration at the end surface was measured. At the point of $\theta=0$, the component vibration $\psi$ was expressed by

$$
\psi_{\theta=0}=A \sin (\omega t+\phi)
$$

where A and $\phi$ are constants. At the point of $\theta, \psi$ is expressed by

$$
\begin{gathered}
\psi_{\theta}=A \sin \left(\omega\left(t-\frac{r \theta}{v}\right)+\phi\right) \\
=A \sin \left(\omega\left(t-\frac{\theta}{\omega}\right)+\phi\right)
\end{gathered}
$$

where $v$ is phase velocity of traveling wave, $r$ is a radius of the stator, $\omega$ is angular velocity. From this equation, time difference $\varphi$ is expressed by

$$
\varphi=\frac{\theta}{\omega}
$$

So the measurement of phase difference which is given as $\varphi \omega$ versus $\theta$ assists the proof of the traveling wave. The result is shown in Fig. 13 which indicates that the traveling wave was excited on the end surface of the stator transducer.

\section{Rotation Result}

The operation test of the motor was carried out using a screw as a rotor. The photograph of the motor is shown in Fig. 16. The cone shaped head screw was used to make the rotor contact with the stator at the inner side edge of the cylinder. The diameter and the weight was $1.7 \mathrm{~mm}$ and $0.05 \mathrm{~g}$. The rotor was put on the stator ,so that the pre load was only the weight of the screw. The rotation speed changed from 0 to $295 \mathrm{rpm}$ with the driving voltage from 20 to $33 \mathrm{Vp}$-p as shown in Fig. 17.

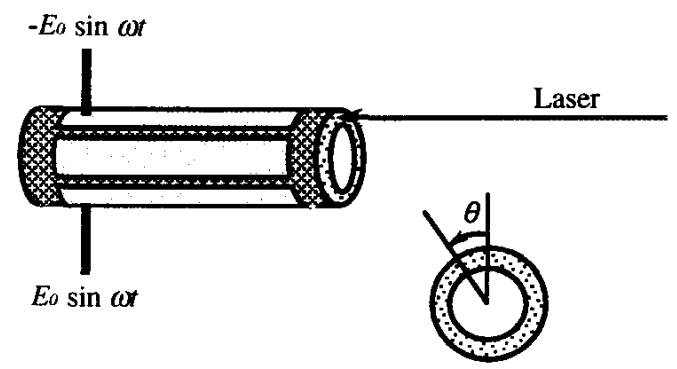

Fig. 13. Measured point of vibration amplitude on side surface.

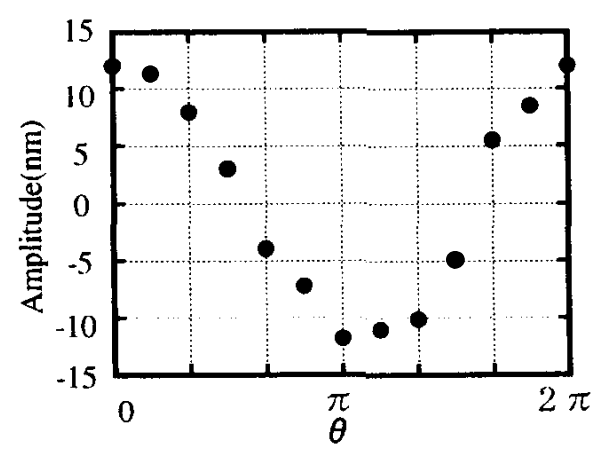

Fig. 14. Amplitude distribution in radial direction on side surface

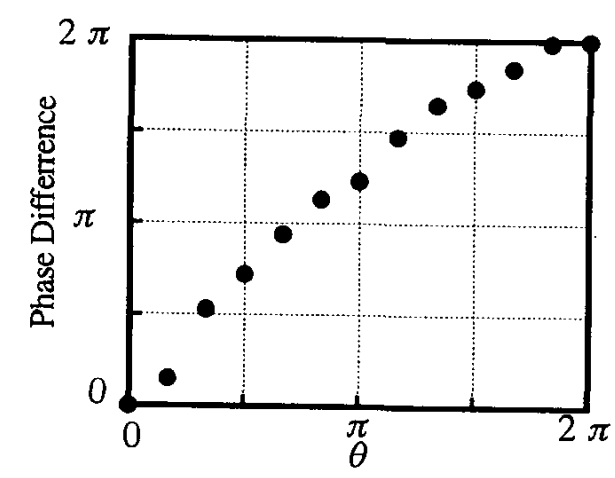

Fig. 15 Phase difference in radial direction on end surface. 


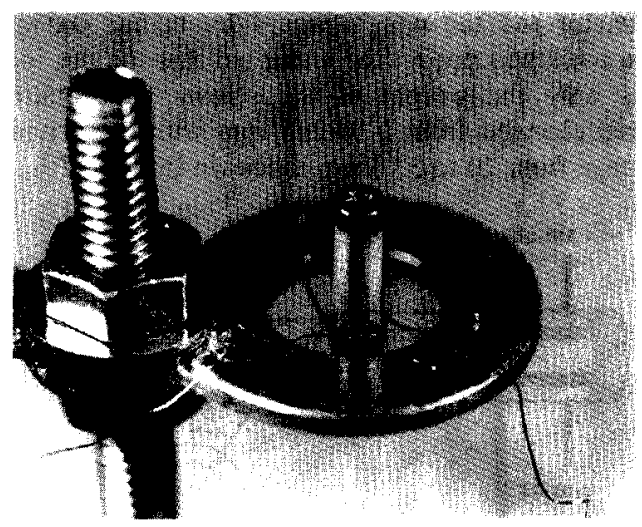

Fig. 16. Photograph of ultrasonic micro motor.

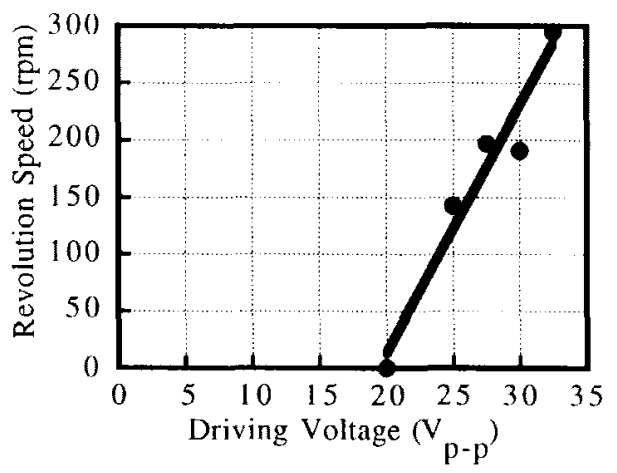

Fig. 17. Revolution Speed versus driving voltage.

\section{CONClusion}

We have proposed a cylindrical micro ultrasonic motor. To fabricate the stator transducer of the motor, the hydrothermal method for the PZT thin film was applied. The PZT film was deposited on the round surface of the titanium pipe. The fabricated transducer worked as expected.

For the next step, the quality of the stator transducer should be improved. The electrodes and the power supply wires caused the vibration loss. The construction should also be improved to get out of the mechanical output power efficiently. The rotor shape, the driving surface and the motor holding would be problems.

At high vibration amplitude, the characteristics of the deposited PZT film should be examined. To estimate the performance of the motor, these information is important.

As the film is thin such as $10 \mu \mathrm{m}$, the actually required driving voltage would be low. Hence 2 or 3 volt will be enough for driving the motor

\section{ACKNOWLeDGMENT}

The authors would like to thank Prof. T. Tsurumi and Dr. Y. Ohba of Tokyo Institute of Technology for assistance on the hydrothermal method of the PZT thin film. This work has bee supported by the Ministry of Education, Science and Culture and the Murata Science Foundation.

\section{REFERENCES}

[1] L. S. Fan, et al. :"IC-processd electrostatic micromotors" Sensors \&Actuators, vol.20, pp.41$48,(1989)$.

[2] W. S. N. Trimmer, et al.," Harmonic Electrostatic Motors" Sensors \& Actuators, vol. 20, pp.17-24,(1989).

[3] A. M. Flynn, L. S. Tavrow, S. F. Bart, R. A. Brooks, D. J. Ehrlich, K. R. Udayakumar and L. E. Cross, "Piezoelectric micromotors for microrobots," in Proc. of 1990 IEEE Ultrason. Symp., pp.11631172, (1990).

[4] G. A. Racine, R. Luthier and N. F. de Rooj, Hybrid ultrasonic micromachined motors," in Proc. of 1993 lEEE MEMS, pp.128-132.

[5] M. Kurosawa, K. Nakamura, T. Okamoto and S. Ueha, "An ultrasonic motor using bending vibration of a short cylinder ," IEEE Trans. on UFFC, vol.36, no.5, pp.517-521, (1989).

[6] I. Okumura, "A designing method of a bartype ultrasonic motor for autofocus lenses," in Proc. of IFToMM-jc. Int. Symp. on Theory of Mach. and Mech., pp.836-841, (1992).

[7] S. P. Timoshenko,et al, "Vibration Problems in engineering Fourth Edition (Chapter five Section twelve)," translated by O.Taniguchi and A. Tamura, [in japanese] (Corona Publishing CO.,LTD. Tokyo Japan 1977)

[8] C. K. Jen, C. Neron, G. Yi and M. Sayer," Acoustic Lenses Employing PZT Thin Film Transducers" Materials Science and Engineering, A122, pp21-25, (1989).

[9] K. Shimomura, T. Tsurumi, Y. Ohba and M. Daimon, "Preparation of lead zirconate titanate thin film by hydrothermal method," Jpn. J. Appl. Phys., vol.30, no.9B, pp.2174-2177, (1991). 